\title{
INTRODUCTION TO THE APPLICATIONS OF EVOLUTIONARY COMPUTATION IN COMPUTER SECURITY AND CRYPTOGRAPHY
}

\author{
PEDRO IsASI AND JULIO C. HERNANDEZ \\ Computer Science Department, Carlos III University, 28911 Legans, Madrid, Spain
}

\section{INTRODUCTION}

Techniques taken from the field of Artificial Intelligence, especially Evolutionary Computation (Genetic Algorithms and Genetic Programming, but also others) are steadily becoming more and more present in the area of computer security, both in network/host security and in the very demanding area of cryptology.

In recent years, many algorithms that take advantage of approaches based on Evolutionary Computation have been proposed, for example, in the design and analysis of a number of new cryptographic primitives, ranging from pseudo-random number generators to block ciphers, in the cryptanalysis of state-of-the-art cryptosystems, and in the detection of network attacking patterns, to name a few.

There is a growing interest from the computer security community toward Evolutionary Computation techniques, as a result of these recent successes, but there still are a number of open problems in the field that should be addressed.

\section{OPEN PROBLEMS}

When using Evolutionary Computation in the field of cryptanalysis, the main issue is how to define fitness functions for the various heuristic methods (say genetic algorithms, simulated annealing, etc.) that avoid the problem of the deceptive fitness landscape. The landscape associated with modern cryptosystems is almost invariably delta-shaped, and straightforwardly applying heuristics to a search in such a space does not necessarily produce results better than random search.

While the classical cryptosystems, nowadays totally broken, have the property that, in general, keys close to the real key produce encryptions/decryptions close to the original ciphertext/plaintext, thus significantly aiding in defining fitness functions (that measure the proximity of a certain given text to English, for example) modern ciphers do not exhibit this property. That is a major problem and the reason behind the relative lack of applications of these heuristics techniques to modern cryptosystems, where while testing a key that has 255 right bits out of 256 (99.61\% correct), the resulting plaintext would appear completely random due to a property that has been named the Avalanche Effect.

In cryptology, two main applications of evolutionary computation techniques have been deeply investigated: the design of good pseudorandom number generators and the design of block cipher s-boxes. The first problem is quite deceptive because all the examined approaches are based in evolving generators whose fitness is measured by observing the pseudorandom properties of part of their output. This is quite a difficult method for measuring 
pseudorandomness because, for being good, the generator should usually have an extremely long period and thus one can only observe a short part of it efficiently. On the other hand, the use of test batteries for randomness as part of the fitness function is an approach that is increasingly recognized as very limited, mainly because randomness is a very elusive concept which has no single, efficiently measurable, definition. That is the reason why these research line is focusing on the last years on developing good pseudorandom numbers for particular applications, where the required properties are well known and easily measurable, abandoning the idealistic idea of creating good pseudorandom generators for all purposes.

The second problem has reached, contrarily to many expectations, a great success. The design of substitution boxes (s-boxes) is a critical phase in the development of a block cipher. When designing a new block cipher, many researchers follow Feistel's recommendations. If so done, the problem is essentially reduced to the design of good s-boxes. Feistel type block ciphers abound, proving the overall scheme, while not optimum, is robust enough. From the ancient DES to the recent CAST-256, many block cipher designers opt for following this scheme due to its simplicity, good properties and robustness, guaranteed by exhaustive testing along the years. So having good algorithms for the automatic generation of s-boxes imply being able of automatically generating good new block ciphers, and that is the reason behind the increasing interest in the field, especially after the many successes obtained by different researchers in the area.

It is foreseeable that in the future, these research trends will be further explored and some of the described open problems will get imaginative and brilliant solutions. It is quite probable, also, that new fields and applications will emerge. The articles presented in this special issue clearly contribute to this effort in both directions.

\section{STATE OF THE ART}

Many researchers in the past have brilliantly worked in the field of artificial intelligence applications to computer security and cryptology. We will very briefly present their most important achievements.

It all started with the pioneer work of Peleg and Rosenfeld (1979). Along these lines it followed the works of Hunter and McKenzie (1983), Carroll and Martin (1986), and King and Bahler (1992), which proposed breaking a simple substitution cipher by means of different relaxation algorithms. Also worth mentioning are the articles by Ganesan and Sherman (1993), which proposed new probability distributions to help in automatically distinguishing between a given language and random text, very useful for key exhaustion, and the paper by Cain and Sherman (1994), where the authors broke the Gifford stream cipher. Ramesh, Athithan, and Thiruvengadam (1993) proposed a very original patterns-based attack against simple substitution ciphers.

Wagner and Bellovin (1994) designed a very efficient plaintext recognizer to allow for speed-ups in brute force attacks. Also in 1994, King showed the first attack on polyalphabetic substitution ciphers, an idea that was later extended and improved by Clark and Dawson (1997) with the help of a parallel genetic algorithm.

We should also mention some other works that had a direct and deep influence over the approaches that many researchers still use today. The work of Spillman et al. (1993), which first introduced genetic algorithms in the cryptanalysis of simple substitution and transposition ciphers; the article by Forsyth and Safavi-Naini (1993) proposed the use of simulated annealing for solving the same problem, and the paper by Jakobsen (1995) showed that simpler and more efficient hill-climbing techniques were also able to break substitution ciphers. 
If one should draw a temporal line between the so-called classical and modern techniques this would be the year 1997, with the paper of Bagnall, McKeown, and RaywardSmith (1997), where the authors presented a ciphertext-only attack over a simplified version of an ENIGMA rotor machine based on the use of a genetic algorithm. Also crucial for the field was the research carried on by the Australian group at QUT, Brisbane, which included the Ph.D. Thesis of Clark, published in 1998. That was a major advance in the area. It introduced the tabu search technique in cryptanalysis, compared different heuristic techniques (genetic algorithms, simulated annealing, and tabu search) at breaking classical cryptosystems and proposed the use of simulated annealing in the cryptanalysis of a certain class of stream ciphers. Millan, Clark, and Dawson additionally proposed a model for the generation of Boolean functions with excellent cryptographic applications, thus starting a very fruitful research line and showing these techniques could also help in cryptography, not only cryptanalysis.

Examples of other successful research directions are, for example, the use of cellular automata. The first papers were those by Wolfram (1986), proposing their use for pseudorandom number generation and for the design of block ciphers. Daamgard also used them in 1991 for designing hash functions, a work which was later cryptanalyzed by Daemen, Govaerts and Vandewalle (1993). Meier and Staffelbach (1991) pointed out many deficiencies of the generator proposed by Wolfram, and later on Daemen, Govaerts and Vandewalle described a new algorithm that solved these problems. Although forgotten during some time, this research line is recently living a renaissance and in the last years many important works have been published in the area, notably by Slipper and Tomassini (1996), who coined the term cellular programming, and more recently by Sheng-Uei and Shu Zhang (2003), which designed a pseudorandom cellular automata based number generator with excellent random properties.

Another very fruitful research area, although not strictly based in the evolutionary computation paradigm, is the application of neural networks to cryptology, where the team was formed by Kinzel. Kanter has produced very interesting results from 2002 that prove neural networks could be used in pseudorandom number generation and public key exchange. Unfortunately, this latter proposal, by far the most interesting, was quickly broken by Klimov, Mityaguine, and Shamir who, curiously, used various techniques based on genetic algorithms and simulated annealing to cryptanalyze it.

More recently, various important contributions to the field have been published by Clark and Jacob, of which we should mention the 2002 article where they propose a totally new way of attacking implementations of cryptographic primitives based on fault injection and timing analysis, which they also show is powerful enough to break the Permuted Perceptron Problem identification schemes by means of simulated annealing. Another interesting research line is that developed by Hernandez et al. (2002), on the analysis of the weaknesses of the modern block cipher TEA, including the automatic construction of distinguishers. Also worth mentioning is the continuation of the work of Bill Millan and Ed Dawson on the generation of good Boolean functions for cryptographic uses. Some of the latest achievements of these works are included in this Special Issue.

\section{ABOUT THIS ISSUE}

This special issue is an exceptional occasion to present the topic and give interested researchers an opportunity to review the current state-of-art of the area by a selection of excellent contributions which show new methodologies, novel applications, and explore promising new directions. 
The articles included are extended and improved versions of the best papers presented to the special session organized by the authors at the 2003 IEEE Congress on Evolutionary Computation. An outstanding panel of reviewers considered these articles the best ones, and also made some comments and suggestions to improve their attractiveness and readability, followed by authors. The final result is this Special Issue.

\section{ACKNOWLEDGMENTS}

We finally want to acknowledge the help of the extraordinary panel of reviewers that worked quite hard in the selection of the papers to be included in this special issue: John Clark, from York University, UK; Bill Millan from Queensland University, Australia; Richard Spillman, from the Pacific Lutheran University at Tacoma, Washington, USA; Tony Bagnall, from the School of Information Systems of the University of East Anglia, UK; Nicholas Hopper from the CMU Computer Science Department, Pittsburgh, USA; Jungwon Kim, from King's College London, UK; Lisa Strite from IBM Raleigh, USA; Carlos A. Coello Coello from CINVESTAV-IPN, México; Jun He, from the University of Birmingham, UK; Hari V. Sahasrabuddhe from the Kanwal Rekhi School of Information Technology at the Indian Institute of Technology, Bombay. Thanks to all of them. Thanks also to the Editors of Computational Intelligence, Randy Goebel, Russell Greiner, and Dekang Lin for their confidence and help, and to Keri Ann Reid, the Editorial Assistant, for her valuable assistance and patience.

\section{REFERENCES}

BAgnall, A. J., G. P. MC KeOWN, and V. J. RAYWARD-SMITH. 1997. The cryptanalysis of a three rotor machine using a genetic algorithm. In Proceedings of the 7th International Conference on Genetics Algorithms ICGA'97. Morgan-Kaufmann.

CAIN, T. R., and A. T. SHERMAN. 1994. How to break Gifford's Cipher. In Proceedings of the ACM Conference on Computer and Communications Security, pp. 198-209.

CArroll, J. M., and S. MARTin. 1986. The automated cryptanalysis of substitution ciphers. Cryptologia, $\mathbf{X}(4): 193-209$.

Clark, A., and E. DAWSON. 1997. A parallel genetic algorithm for cryptanalysis of the polyalphabetic substitution cipher. Cryptologia, 21(2):129-138.

Clark, A. J. 1998. Optimization Heuristics for Cryptology. Ph.D. Thesis, Information Security Research Center, Faculty of Information Technology, Queensland University of Technology.

ClARK, J. A., and J. L. JACOB. 2002. Fault injection and a timing channel on an analysis technique. In Proceedings of Eurocrypt 2002, pp. 181-197.

DAemen, J., R. GovaerTs, and J. VANDEWAlLE. 1993. A framework for the design of one-way hash functions including cryptanalysis of Damgaard's one way function based on cellular automaton. Advances in Cryptology: Proceedings of Asiacrypt'91, LNCS v. 739, pp. 82-96.

FORSYTH, W. S., and R. SAFAVI-NAINI. 1993. Automated cryptanalysis of substitution ciphers. Cryptologia, XVII(4):407-418.

GANESAN, R., and A. T. SHERMAN. 1993. Statistical Techniques for Language Recognition: An introduction and guide for cryptanalysts. Cryptologia, XVII(4):321-366.

Hernandez, J., J. M. Sierra, P. ISASI, and A. RIBAgOrdA. 2002. Genetic cryptoanalysis of two rounds TEA. Lecture Notes in Computer Science, 2331:1024-1031.

Hunter, D. G. N., and A. R. MCKEnZIE. 1983. Experiments with relaxation algorithms for breaking simple substitution ciphers. The Computer Journal, 26(1):68-71. 
KANTER I., W. KINZEL, and E. KANTER. 2002. Secure exchange of information by synchronization of neural networks. Europhyses Letters, 57:141.

KING, J. C. 1994. An algorithm for the complete cryptanalysis of periodic polyalphabetic substitution ciphers. Cryptologia, XVIII(4):332-355.

KING, J. C., and D. R. BAHLER. 1992. An implementation of probabilistic relaxation in the cryptanalysis of simple substitution ciphers. Cryptologia, XVI(3):215-225.

MeIER, W., and O. Staffelbach. 1991. Analysis of Pseudo Random Sequences Generated by Cellular Automata. Advances in Cryptology. In Proceedings of Eurocrypt'91, Springer-Verlag LNCS v. 547, pp. 186-199.

PELEG, S., and A. ROSENFELD. 1979. Breaking substitution ciphers using a relaxation algorithm. Communications of the ACM, 22(11):598-605.

RAMESh, R. S., G. ATHITHAN, and K. ThIRUVENGADAM. 1993. An automated approach to solve simple substitution ciphers. Cryptologia, XVII(2):202-218.

SLIPPER, M., and M. TOMASSINI. 1996. Co-evolving parallel random number generators. In Proceedings of the Parallel problem solving from Nature-PPSN IV, pp. 950-959. Springer-Verlag.

SPILlma, R., M. JANSSEN, B. NELSON, and M. KEPNER. 1993. Use of a genetic algorithm in the cryptanalysis of simple substitution ciphers. Cryptologia, XVII(1):31-44.

SHENG-UEI, and S. ZHANG. 2003. An evolutionary approach to the design of controllable cellular automata structure for random number generation. IEEE Transactions on Evolutionary Computation, 7(1).

WAGNER, D., and S. M. BELLOVIN. 1994. A programmable plaintext recognizer. Unpublished manuscript. Available at http://www.cs.berkeley.edu/ daw/papers/recog.ps.

WOLFRAM, S. 1986. Random sequence generation by cellular automata. Advances in Applied Mathematics, 7:123. 